\title{
Understanding the Cognitive Processes involved in Technological Entrepreneurial Opportunity Recognition
}

\author{
P.Bhaskara Reddy, R.Praven Reddy and S.Madhu \\ pbhaskarareddy@rediffmail.com; rpreddy82@gmail.com; salumari@gmail.com
}

\begin{abstract}
:
In the state-of-the art An Agenda for Future Research, Corbett and McMullen (2011) summarize the current state of the research in the following words - "Despite its many achievements, learning at the connection of free enterprise and cognition has focused primarily on the consequences of what happens when an industrialist profit from various cognitive characteristics, resources, or other dispositions. As such, cognitive study in free enterprise continues to experience from narrow theoretical articulations and weak theoretical practicalities that lower its role to the managerial sciences."According to Santos and Eisenhardt (2004)" entrepreneurs categorize innovative opportunities for the conception of worth, and construct a market around those opportunities" triumphant chance appreciation leads to successful startups and leads to the vibrancy of economy. However, very little work has been done in this field (Mitchell et al., 2014).

Key words: Industrial, Authority, Approach, Perceptive, Gratitude, Cognition, Experimental, Inspiration, Proficiency

\section{Technical Details}

The objective is to understand the Cognitive Processes involved in Technological Entrepreneurial Opportunity Recognition. This step involves creating a model with various skill sets that could influence opportunity recognition. Also, the modeling involves identifying the interrelationships between these skills. The second objective establishes the baseline data for the Indian technology entrepreneurs and compares it with their counterparts in USA. This benchmarking can help in establishing aspirational goals and also transforming our education system.
\end{abstract}

\section{Introduction}

In the state-of-the artarticle "The Cognitive Perspective in Entrepreneurship: An Agenda for Future Research," Grégoire, Corbett and McMullen (2011) summarize the current state of the research in the following words - "Despite its many achievements, scholarship at the intersection of entrepreneurship and cognition has focused primarily on the consequences of what happens when an entrepreneur benefits from various cognitive characteristics, resources, or other dispositions. As such, cognitive research in entrepreneurship continues to suffer from narrow theoretical articulations and weak conceptual foundations that lessen its contribution to the managerial sciences."According to Santos and Eisenhardt (2004)"entrepreneurs perceive new opportunities for the creation of value, and construct a market 
P.Bhaskara Reddy, R.Praven Reddy and S.Madhu; Understanding the Cognitive Processes involved in Technological Entrepreneurial Opportunity Recognition, Transactions on Machine Learning and Artificial Intelligence,

Volume 3 No 5 October, (2015); pp: 1-9

around those opportunities" Successful opportunity recognition leads to successful start-ups and leads to the vibrancy of economy. However, very little work has been done in this field (Mitchell et al., 2014).

\section{Origin of the Proposal}

Opportunity recognition is considered as a key cognitive skill that distinguishes an entrepreneur from the rest and is the beginning stage of the entrepreneurial journey (Christensen et al. 1994; Gaglio 1997; Knowlton, 1997; Shane and Venkataraman, 2000).Gaglio and Katz (2001:95) even describe its importance as "understanding the opportunity identification process represents one of the core intellectual questions for the domain of entrepreneurship" Baron (2006) identified three critical elements for the opportunity identification as actively seeking or searching for opportunities, attentiveness or alertness to potential opportunities and previous knowledge, which includes customers, market, technology and industry.

\section{Definition of the Problem}

To date, we have very little or no information about the cognitive processes involved in the technical opportunity recognition. As a result, we may be ineffectively training our future engineers and hampering their entrepreneurial mindset. Our goal is to understand technical opportunity recognition process and benchmark the abilities of Indian entrepreneurs. This study will be the first study to comprehensively examine the topic of opportunity recognition and establish the baseline information for the Indian entrepreneurs.

\section{Objective}

The objective is to understand the Cognitive Processes involved in Technological Entrepreneurial Opportunity Recognition. This step involves creating a model with various skill sets that could influence opportunity recognition. Also, the modeling involves identifying the interrelationships between these skills. The second objective establishes the baseline data for the Indian technology entrepreneurs and compares it with their counterparts in USA. This benchmarking can help in establishing aspirational goals and also transforming our education system.

\section{Review of Status of Research and Development in the Subject}

As Albert Einstein famously noted "Innovation is not a product of logical thought, although the result is tied to logical structure." As academicians, we often try to put a systematic procedure around this nonlinear process. In fact, we teach our engineering and business students systematic search models for opportunity recognition (Fiet, 2002). However, it is very well noted that entrepreneurs don't follow systematic search or heuristic methods (Shaver \& Scott, 1991 and Busenitz\& Barney, 1997). In a similar open-ended problem solving domain, Brooks (2003) notes that "...the rational models of the design process ...is dead wrong and seriously misleading." In fact the models donot capture the process adopted by experts and therefore, results in "bizarre" results.Kirkner (1973 \& 1979) introduced the concept of entrepreneurial alertness. It refers to the cognitive ability to identify opportunities without consciously searching for them. According to Kirzner, alter individuals experience an "aha" moment, which provides a different point of view and helps him/her to identify the opportunity in a very nonlinear fashion.

Groves (2011) empirically studied entrepreneurial cognition of entrepreneurs and concluded that the successful entrepreneurs have a versatile balance of the nonlinear thinking with more rational linear thinking. This landmark study finds differences in 219 professionals (not students), which includes 39 
entrepreneurs, and uses the Linear and Nonlinear Thinking Stile Profile (LNTSP) instrument developed by Vance et al. (2007). This study is relevant to this proposal as it uses the same instrument to see the balance in Indian entrepreneurs.

DeTienne and Chandler (2004) empirically study the influence of entrepreneurial classroom using a Solomon Four-Group Designed experiment and propensity to innovate using Kirton Adaptor Innovator inventory (KIA) instrument. This particular study is very relevant to this proposal as it provides similar benchmarking data for young entrepreneurs in USA.

Finally, Pistrui (2013) uses a well-recognized survey instrument by Target Training Institute (TTI) to understand entrepreneurial mindset in engineering student in USA. The instrument creates a 62-page report that provides detailed qualitative and quantitative insight into behavior, motivation and skill. The survey results includes:

a) Insight into natural and adapted behavior in terms of DISC (Dominance, Influence, Steadiness, Compliance). According to TTI, DISC is "is the language of how we act-i.e. our behavior. Research has consistently shown that behavioral characteristics can be grouped together into four quadrants, or styles. People with similar styles tend to exhibit specific types of behaviors common to that style - this is not acting. A person's behavior is a necessary and integral part of who they are. In other words, much of our behavior comes from "nature" (inherent), and much comes from "nurture" (our upbringing). The DISC model merely analyzes behavioral styles; that is, a person's manner of doing things." The instrument provide both qualitative and quantitative analysis as shown in the figure below

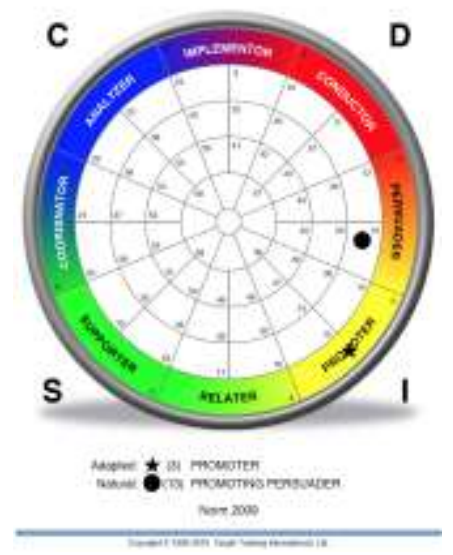

Sample DISC Wheel

b) Motivators are factors that drive our behavior or in other words, the motivators tell why we do things. The six values or motivators are

1. Theoretical is drive for acquiring new knowledge

2. Utilitarian is the drive for creating a value proposition that solves peoples problems.

3. Aesthetic is the drive for harmony in form.

4. Social/altruistic is the drive for helping the society

5. Individualistic is the drive for control and power.

6. Traditional is the drive for maintaining the order.

sample results are shown in the figure below. 
P.Bhaskara Reddy, R.Praven Reddy and S.Madhu; Understanding the Cognitive Processes involved in Technological Entrepreneurial Opportunity Recognition, Transactions on Machine Learning and Artificial Intelligence,

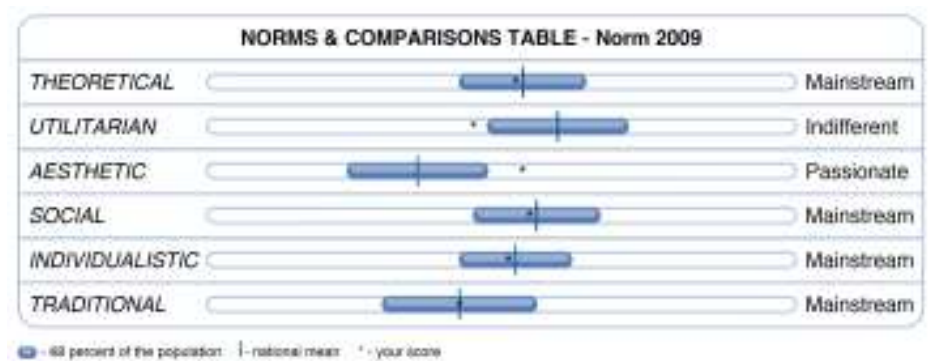

Sample motivator

c) The instrument also ranks the core skills of the individual. Crawford et al. (2011) associated the seven professional skill clusters with the right brain thinking -

1. Experiences

2. Team Skills

3. Communication Skills

4. Leadership Skills

5. Decision Making/Problem Solving Skills

6. Self-Management Skills

7. Professionalism Skills

TTI survey expands them into lower level skills as shown in the sample data below.

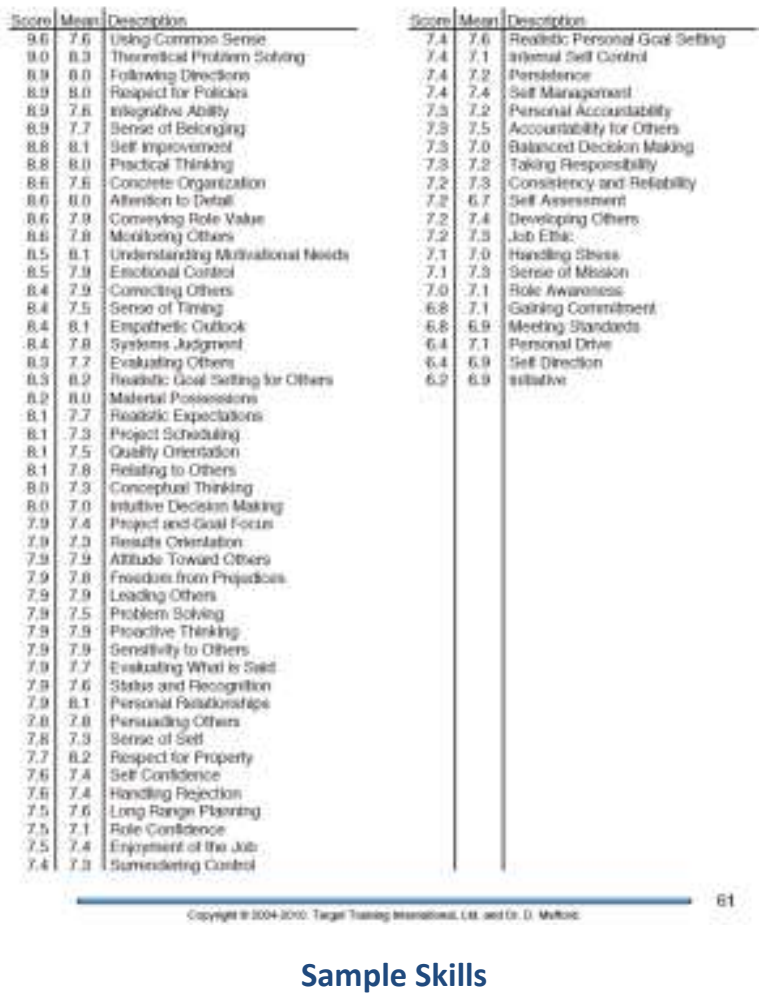

Pistrui et al. (2012) investigated the relationship between TTI assessment data and Entrepreneurial mindset based on a major survey of approximately 5000 undergraduate students and 300 practicing entrepreneurs. This is the most comprehensive empirical study performed in the area of engineering 
entrepreneurship. Once again, we plan to use this instrument to independently validate the model and also benchmark our engineering entrepreneurs.

As opposed to previous three empirical studies, Corbett (2007) based on an experimental task studied 380 technology professionals to understand how opportunities are identified based on the learning (acquiring and transforming information and experience). This study is important as it provides benchmark data and also provides another valid instrument to understand the opportunity recognition process.

\section{International status}

The primary studycomes from the Kern Entrepreneurship Education Network - a group of private higher education institutions in USA who are reforming engineering education to incorporate entrepreneurial mindset (Pistrui, 2013). The other groups studying include Rensselaer Polytechnic Institute and now Babson College which is ranked no 1 in entrepreneurship education (Corbett, 2005; Corbett et al. 2014) and Colorado State University (DeTienne and Chandler, 2004).

\section{National status}

Garud and Prasad (2013) from IIM Bangalore describe cognition in the R\&D management in Indian hi-tech firms. Kundu and Rani (2008) conducted entrepreneurial attitude and orientation depending on gender and background in the Indian airforce trainees. A Conceptual Framework.Gangaiah and Viswanath (2014) studied the impact of management education in developing entrepreneurial aspiration and attitudes in the Indian context with business students. This research is centered on the problems in the management education. However, very little is done in the area of cognitive science as applied to technology opportunity recognition.

\section{Importance of the Proposed Project in the Context of Current Status}

Most studies of the opportunity recognition are in the area of business and not based on cognitive understanding. This study will provide an insight into how technology entrepreneurs think in India and also contrasts them with the engineering entrepreneurs. This study also benchmarks the skills of our entrepreneurs with the counterparts in USA.

The study helps to better understand the technology opportunity recognition process based on cognitive science. This in turn can help us can profoundly change the way we teach opportunity recognition as a subject and also in different courses in the engineering education. It also helps to identify the strengths and weaknesses of our engineering entrepreneurs so that we can systematically address them.

\section{Methodology}

The first part of the proposal requires creating an opportunity recognition model from the existing literature. During this phase, extensive literature review will be carried out to understand the opportunity recognition process particularly in engineering domain. Most models are very similar framework with four or five basic steps (shown in the figure below) with different levels of details. However, the focus of this particular study will be in understanding cognitive traits that play a crucial role in the process. The model will capture the current thinking in the domain of cognitive science and opportunity recognition.

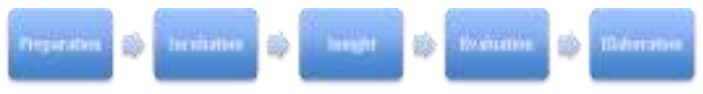

Basic steps in the opportunity recognition process 
P.Bhaskara Reddy, R.Praven Reddy and S.Madhu; Understanding the Cognitive Processes involved in Technological Entrepreneurial Opportunity Recognition, Transactions on Machine Learning and Artificial Intelligence,

Volume 3 No 5 October, (2015); pp: 1-9

Once the model is complete, the task of collecting empirical data to fine-tune the model begins. During this task, we adapt the three empirical studies to include an emphasis on technology opportunity recognition. For these studies, the principal investigator will recruit 250 entrepreneurs from non-technical areas and 250 entrepreneurs with technical background. These participants will be given the Solomon Four-Group Designed experiment, Kirton Adaptor Innovator inventory (KIA) instrument, andNonlinear Thinking Stile Profile (LNTSP) instrument. These instruments are used by Groves (2011) and DeTienne and Chandler (2004). Thus, these experiments will provide a bench mark data as applied to Indian ecosystem as well as provide additional insights as we are looking into the technology domain. Further, a select number of total sample (a total of 50 participants) will be given both TTI survey. Consistent with Pistrui study (2013), the Structural Equation Modeling or SEM techniquewhich is useful understanding and evaluating the relationshipbetween human behavior and human motivation (Wallgren and Hanse, 2007; Williams et al., 2003) and Cronbach's Alpha, an accepted measure of internal consistency or reliability will be used to perform factor analysis of a combined model (Cronbach, 1951).

These select group will also be asked to repeat Corbett's experiment with the additional verbal protocol analysis method. Verbal protocol analysis is a think aloud method where participants or subjects verbalize their thought processes while performing the design task. After the task is completed, these audio/video sessions are transcribed and analyzed to gain insights into the thought process thereby answering the research question. This type of analysis has been accepted as a valid research method (Ericsson, 1980). The protocol analysis method will be to replicate Corbett's experiment.

In the experiment, Corbett asks participants to identify new opportunities for the Bluetooth technology. The actual instructions are shown in the figure below. Note that in additional to Corbett's experiment instructions will be modified to asking participants to verbalize the thoughts. These verbal thoughts are both audio and video taped. It is typically acceptable to perform the protocol analysis on a much smaller set of participants. Also, they will be asked to perform a second task "design a innovative planter for people living in apartments for gardening." The task will require the participants to sketch as engineers are visual thinkers and Cobett's experiment doesn't capture the visual thinking.

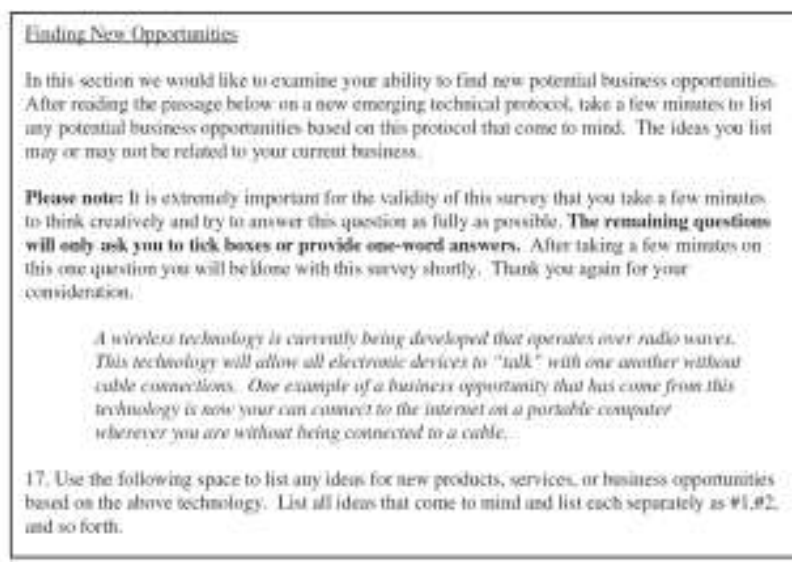

Corbett's experiment

These large quantitative empirical data coupled with detailed qualitative data obtained through the protocol analysis will help to fine tune the model, identify interrelationship between the various parameters, and spots trends in people who are good in opportunity recognition compared to others. It 
also provides insight into the development or a lack development of opportunity recognition skills during the course of engineering study. This information will be used to fine-tune the model with quantitative relationship between various cognitive skills.

Once, the model is completely developed, a large-scale study of 5000 participants will be undertaken. The verbal protocol study will be on a smaller sample size as it is time consuming to analyze the data and TTI survey will also be on a smaller size as it is expensive. The large-scale study will be used to validate the opportunity recognition model.

\section{Organization of Work Elements}

In the state-of-the art article "The Cognitive Perspective in Entrepreneurship: An Agenda for Future Research," Grégoire,Corbett and McMullen (2011)summarize the current state of the research in the following words - "Despite its many achievements, scholarship at the intersection of entrepreneurship and cognition has focused primarily on the consequences of what happens when an entrepreneur benefits from various cognitive characteristics, resources, or other dispositions. Assuch, cognitive research in entrepreneurship continues to suffer from narrow theoretical articulations and weak conceptual foundations that lessen its contribution to the managerial sciences."According to Santos and Eisenhardt (2004)"entrepreneurs perceive new opportunities for the creation of value, and construct a market around those opportunities" Successful opportunity recognition leads to successful start-ups and leads to the vibrancy of economy. However, very little work has been done in this field (Mitchell et al., 2014).

1. The objective is to understanding the Cognitive Processes involved in Technological Entrepreneurial Opportunity Recognition. This step involves creating a model with various skill sets that could influence opportunity recognition. Also, the modeling involves identifying the interrelationships between these skills.

2. The second objective establishes the baseline data for the Indian technology entrepreneurs and compares it with their counterparts in USA. This benchmarking can help in establishing aspirational goals and also transforming our education system.

\section{REFERENCES}

[1] Baron, R. A., \& Ensley, M. D. (2006). Opportunity recognition as the detection of meaningful patterns: Evidence from comparisons of novice and experienced entrepreneurs. Management science, 52(9), 1331-1344.

[2] Brooks, F., 2003. Is There a Design of Design? NSF Workshop on Science of Design: Software Intensive Systems.

[3] Busenitz, L. W., \& Barney, J. B. (1997). Differences between entrepreneurs and managers in large organizations: Biases and heuristics in strategic decision-making. Journal of business venturing, 12(1), 9-30.

[4] Christensen, P. S., Madsen, O. O., \& Peterson, R. (1994). Conceptualizing entrepreneurial opportunity identification. Marketing and Entrepreneurship: Research Ideas and Opportunities. Ed. G. Hills. Westport, CT: Greenwood Press. 
P.Bhaskara Reddy, R.Praven Reddy and S.Madhu; Understanding the Cognitive Processes involved in Technological

Entrepreneurial Opportunity Recognition, Transactions on Machine Learning and Artificial Intelligence,

Volume 3 No 5 October, (2015); pp: 1-9

[5] Corbett, A. C. (2007). Learning asymmetries and the discovery of entrepreneurial opportunities. Journal of Business Venturing, 22(1), 97-118.

[6] Corbett, A. C., Siegel, D. S., \& Katz, J. A. (Eds.). (2014). Academic Entrepreneurship: Creating an Entrepreneurial Ecosystem (Vol. 16). Emerald Group Publishing.

[7] Cronbach, L. J. (1951). Coefficient alpha and the internal structure of tests.psychometrika, 16(3), 297-334.

[8] Crawford, P., Lang, S., Fink, W., Dalton, R., and Fielitz, L. (2011). Comparative Analysis of Soft Skills: What is important for New Graduates. Michigan State University and the University Industry Consortium. 1-24.

[9] DeTienne, D. R., \& Chandler, G. N. (2004). Opportunity identification and its role in the entrepreneurial classroom: A pedagogical approach and empirical test. Academy of Management Learning \& Education, 3(3), 242-257.

[10] Ericsson, K. A., \& Simon, H. A. (1980). Verbal reports as data. Psychological review, 87(3), 215.

[11] Gaglio, C. M. (1997). Opportunity identification: Review, critique and suggested research directions. Advances in entrepreneurship, firm emergence and growth, 3, 139-202.

[12] Gaglio, C. M., \& Katz, J. A. (2001). The psychological basis of opportunity identification: Entrepreneurial alertness. Small business economics, 16(2), 95-111.

[13] Gangaiah, B., \&Viswanath, J. (2014). Impact of Indian Management Education in Developing Entrepreneurial Aspirations and Attitudes among Management Students.Asia Pacific Journal of Research Vol: I Issue I.

[14] Garud, N., \& Prasad, L. Managerial Cognition in R\&D Processes Within Indian hi-tech firms: A Conceptual Framework.

[15] Grégoire, D. A., Corbett, A. C., \& McMullen, J. S. (2011). The cognitive perspective in entrepreneurship: an agenda for future research. Journal of Management Studies, 48(6), 14431477.

[16] Groves, K., Vance, C., \& Choi, D. (2011). Examining entrepreneurial cognition: an occupational analysis of balanced linear and nonlinear thinking and entrepreneurship success. Journal of Small Business Management, 49(3), 438-466.

[17] Kirzner Israel, M. (1973).Competition and entrepreneurship.

[18] Kirzner Israel, M. (1979), Perception, Opportunity and Profit. Chicago. 
[19] Knowlton, B. (1997). Declarative and nondeclarative knowledge: Insights from cognitive neuroscience. Knowledge, concepts, and categories, 215-246.

[20] Kundu, S. C., \& Rani, S. (2008). Human resources' entrepreneurial attitude orientation by gender and background: a study of Indian Air Force trainees. International Journal of Management and Enterprise Development, 5(1), 77-101.

[21] Mitchell, R. K., Busenitz, L. W., Bird, B., Marie Gaglio, C., McMullen, J. S., Morse, E. A., \& Smith, J. B. (2007). The central question in entrepreneurial cognition research. Entrepreneurship Theory and Practice, 31(1), 1-27.

[22] Mitchell, J. R., Mitchell, R. K., \& Randolph-Seng, B. (Eds.). (2014). Handbook of entrepreneurial cognition. Edward Elgar Publishing.

[23] Pistrui, D., Bonnstetter, R., Bonnstetter, B. J., \& Fry, C. C. (2011). Creating, Educating and Assessing a New Class of Entrepreneurial Minded Engineers.The Journal of Engineering Entrepreneurship, 2(2), 1-14.

[24] Pistrui, D., Layer, J. K., \& Dietrich, S. (2012). Mapping the behaviors, motives and professional competencies of entrepreneurially minded engineers in theory and practice: an empirical investigation. In Proceedings American Society for Engineering Education 2012 Annual Conference.

[25] Shane, S., \&Venkataraman, S. (2000).The promise of entrepreneurship as a field of research.Academy of management review, 25(1), 217-226.

[26] Shaver, K. G., \& Scott, L. R. (1991). Person, process, choice: The psychology of new venture creation. Entrepreneurship theory and practice, 16(2), 23-45.

[27] Vance, C. M., Groves, K. S., Paik, Y., \& Kindler, H. (2007). Understanding and measuring linearnonlinear thinking style for enhanced management education and professional practice. Academy of Management Learning \& Education, 6(2), 167-185.

[28] Wallgren, L. G. and Hanse, J. J. (2007). Job characteristics, motivators, and stress among information technology consultants: A structural equation modeling approach. International Journal of Industrial Ergonomics, 37(1): 51-59.

[29] Williams, L.J., Edwards, J.R., and Vandenberg, R.J. (2003). Recent advances in causal modeling methods for organizational and management research. Journal of Management, 29(6): 903-936. 